\section{(A) Check for updates}

Cite this: Analyst, 2020, 145, 3686

\title{
Quantification of protein glycation using vibrational spectroscopy $\dagger$
}

\author{
Bethan S. McAvan, (ID a Aidan P. France, ${ }^{a}$ Bruno Bellina, (D) ${ }^{a}$ Perdita E. Barran, (DD ${ }^{a}$ \\ Royston Goodacre (D) ${ }^{b}$ and Andrew J. Doig (D)*c
}

\begin{abstract}
Glycation is a protein modification prevalent in the progression of diseases such as Diabetes and Alzheimer's, as well as a byproduct of therapeutic protein expression, notably for monoclonal antibodies (mAbs). Quantification of glycated protein is thus advantageous in both assessing the advancement of disease diagnosis and for quality control of protein therapeutics. Vibrational spectroscopy has been highlighted as a technique that can easily be modified for rapid analysis of the glycation state of proteins, and requires minimal sample preparation. Glycated samples of lysozyme and albumin were synthesised by incubation with $0.5 \mathrm{M}$ glucose for 30 days. Here we show that both FTIR-ATR and Raman spectroscopy are able to distinguish between glycated and non-glycated proteins. Principal component analysis (PCA) was used to show separation between control and glycated samples. Loadings plots found specific peaks that accounted for the variation - notably a peak at $1027 \mathrm{~cm}^{-1}$ for FTIR-ATR. In Raman spectroscopy, PCA emphasised peaks at $1040 \mathrm{~cm}^{-1}$ and $1121 \mathrm{~cm}^{-1}$. Therefore, both FTIR-ATR and Raman spectroscopy found changes in peak intensities and wavenumbers within the sugar $\mathrm{C}-\mathrm{O} / \mathrm{C}-\mathrm{C} / \mathrm{C}-\mathrm{N}$ region $\left(1200-800 \mathrm{~cm}^{-1}\right)$. For quantification of the level of glycation of lysozyme, partial least squares regression (PLSR), with statistical validation, was employed to analyse Raman spectra from solution samples containing $0-100 \%$ glycated lysozyme, generating a robust model with $R^{2}$ of 0.99 . We therefore show the scope and potential of Raman spectroscopy as a high throughput quantification method for glycated proteins in solution that could be applied in disease diagnostics, as well as therapeutic protein quality control.
\end{abstract}

Received 18th November 2019 Accepted 22nd January 2020

DOI: 10.1039/c9an02318f

rsc.li/analyst react with susceptible amine groups of amino acids resulting in the formation of a Schiff base. The Schiff base then undergoes spontaneous rearrangements to form the Amadori product. The Amadori product is capable of degrading through many complex pathways. ${ }^{8}$

In vivo the most problematic resultant structures are known as advanced glycation end products (AGEs) which are formed due to reaction of the glycated proteins and glucose in the blood. High levels of AGEs have been linked with many degenerative diseases and can cause vascular problems. ${ }^{9}$ In diabetic patients, abnormally high blood glucose concentrations cause glycation of plasma proteins, such as hemoglobin, giving HbA1c as the most common form. HbA1c is now used to diagnose diabetes, as the amount of glycation reflects the long term concentration of glucose in the blood. Many diabetes studies on treatment and diagnosis now also look at other plasma proteins, such as albumin and IgG, which can also be glycated. $^{8,10}$ Patients with Alzheimer's disease have high accumulation of AGEs on top of the increased levels of AGEs that occur with natural aging. Research for Alzheimer's diagnostics now focuses on glycated products in the brain tissues and cerebrospinal fluid (CSF). Therefore glycation products are have a wide scope as biomarkers for diagnostic purposes. ${ }^{11}$ 


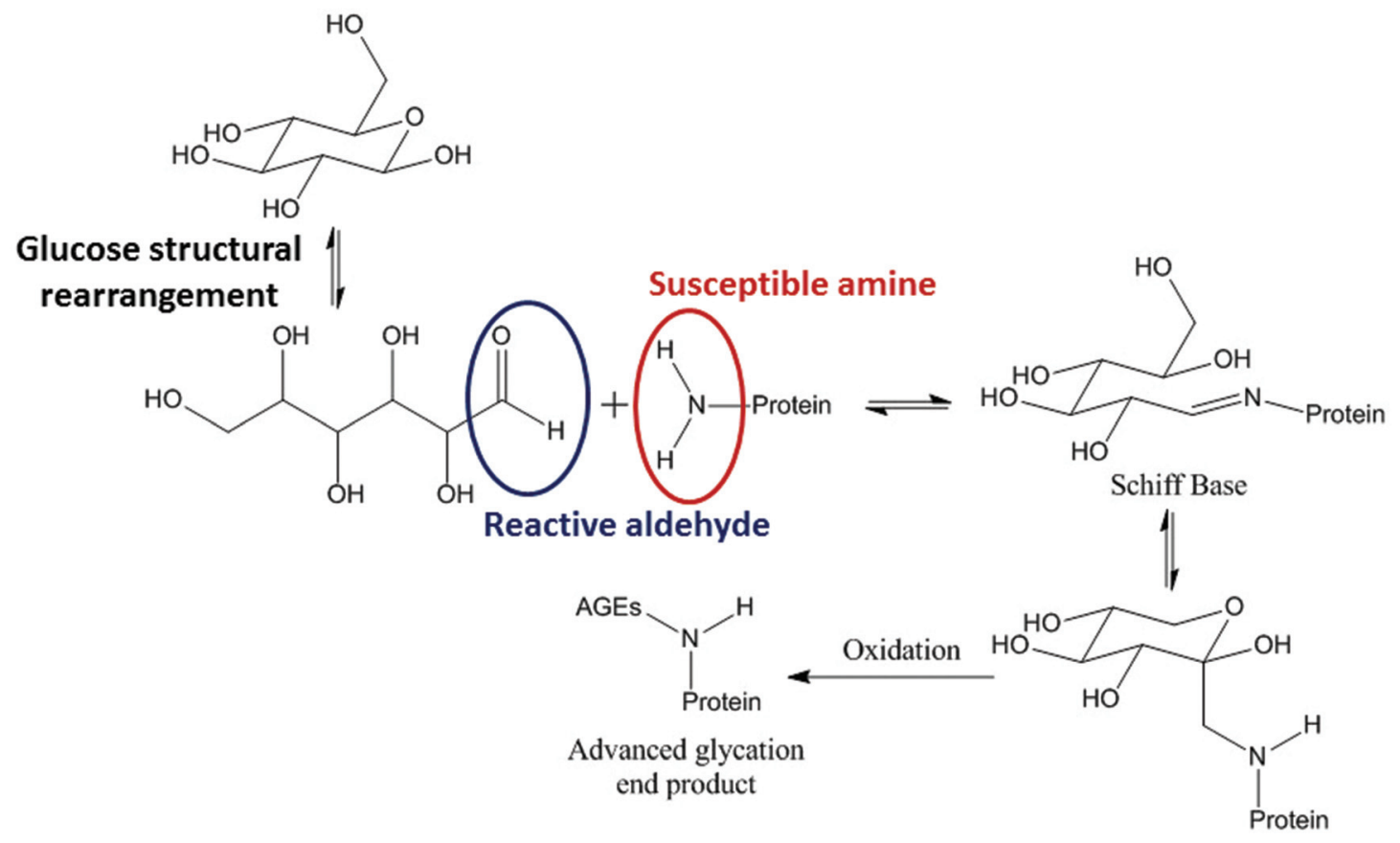

Fig. 1 Reaction scheme showing the general process of glycation in proteins.

The pharmaceutical industry has been increasingly turning to biologically based drugs in the treatment of autoimmune and difficult to treat diseases. Monoclonal antibodies (mAbs) are one of the most common types of biotherapeutic. Glycation has been proven to be problematic during therapeutic expression. mAbs are often expressed using Chinese hamster ovary (CHO) systems in which glucose or other sugars are used as the primary feed source to drive production of the proteins. For example, the mAb Rhumab manufactured by Genentech and Roche, has been found to have between $40-60 \%$ glycation when expressed in specific CHO cell lines. ${ }^{12}$ This high glycation level is thought to be due to a modified Lys in the mAb light chain. Previous literature into the effects that glycation has on both the structure and function of proteins, specifically mAbs, have mixed outcomes. It has been reported that glycation in mAbs is often at $\sim 5 \%$ or less and seems to have minimal effects on binding efficacy and producing immunogenic responses from $\mathrm{T}$ cells in vitro. ${ }^{13}$ However, others report that minor structural deviations in sugar patterns can lead to diminished antibody functionality and batch heterogeneity, especially if the glycation is prevalent in the antigen binding domain of the mAb. ${ }^{5,14,15}$ Irrespective of the effects on structure and function, the International Council for Harmonisation of Technical Requirements for Pharmaceuticals for Human Use (ICH), European Medicines Agency (EMA) and The Food and Drug Administration (FDA) still require glycation levels to be reported as standard quality control for therapeutic proteins. ${ }^{16-18}$

Analysis and quantification of glycation currently relies on laborious and expertise intensive methods such as mass spectrometry (MS) alone or combined with peptide mapping that includes targeted enzymatic digestion of protein and MS to determine the mass of fragments. ${ }^{19}$ Alternatively, boronate columns (BAC) with affinity for glycated proteins can be used in native conditions. These are limited, however, as they have similar affinities no matter how many times the protein is glycated. ${ }^{20-22}$ In this paper, we present vibrational spectroscopy as an analytical method for the quantification of glycation in a model protein, lysozyme. Lysozyme has 6 Lys residues and the $\mathrm{N}$-terminus, resulting in 7 potential glycation sites. To our knowledge, this is the first reported quantification of protein glycation in solution using Raman spectroscopy. ${ }^{23-26}$ Raman spectroscopy is inherently easier to incorporate into bio-manufacturing than other widely used analytical methods such as MS, peptide mapping, or the use of columns. Furthermore, Raman spectroscopy has minimal contribution of water bands and spectroscopic information can be obtained without the requirement for a sample to be taken from a fermentation. It can therefore be used in situ. ${ }^{27-29}$ This paper therefore provides proof-of-concept for using Raman spectroscopy as a method for rapid quantification of glycation and for future development of in-line quality control of protein therapeutic manufacture. It has wider applications in disease diagnosis using glycated proteins implicated in diabetes and Alzheimer's.

\section{Experimental section}

Full experimental details are provided in the ESI. $\dagger$

\section{Materials and chemicals}

Recombinant human lysozyme, $\geq 100000$ units per mg protein (Lysobac) was obtained from Sigma Aldrich (Missouri, US) as a 
lyophilised powder. D-(+)-Glucose $\geq 99.5 \%$ anhydrous was purchased from Thermo Fischer. Recombinant human albumin, was purchased from Sigma Aldrich as a lyophilised powder. Amicon Ultra $0.5 \mathrm{~mL}$ centrifugal filter units, $10 \mathrm{kDa}$ cut off were used for buffer exchange. For the quantification, lysozyme and albumin were buffer exchanged to remove free glucose using Centripure P100 columns (Emp Biotech $\mathrm{GmbH}$ ) before incubation. Sartorius Stedim Biotech ministart $0.2 \mu \mathrm{m}$ syringe filters were used to filter the incubation buffers.

\section{Sample preparation}

Glycation of lysozyme. All incubations were carried out under sterile conditions. Lysozyme was prepared as a $5 \mathrm{mg}$ $\mathrm{mL}^{-1}$ solution in $(28 \mathrm{~mL}) 25 \mathrm{mM}$ MOPS, $115 \mathrm{mM} \mathrm{NaCl}$, adjusted to $\mathrm{pH}$ 7.4. This was subsequently divided into 2 equal volumes of $14 \mathrm{~mL}$ to create two groups of control and glucose incubations. To the glucose incubation group, glucose was added to create an overall sugar concentration of $0.5 \mathrm{M}$. Both groups were filter sterilised using syringe filters and aliquoted into 7 falcon tubes each. All samples were statically incubated at $37^{\circ} \mathrm{C}$. Samples from each group were removed at days 1,2 , $5,7,14,21$, and 30. After removal from incubation, the samples underwent buffer exchange to Millipore grade $\mathrm{H}_{2} \mathrm{O}$ using Amicon centrifugal filters. The samples were then flash frozen, freeze dried overnight and stored at $-20{ }^{\circ} \mathrm{C}$ until analysis.

Glycation of albumin. Albumin was prepared as a $5 \mathrm{mg}$ $\mathrm{mL}^{-1}$ solution in $(30 \mathrm{~mL}) 25 \mathrm{mM}$ MOPS, $115 \mathrm{mM} \mathrm{NaCl}$, adjusted to $\mathrm{pH}$ 7.4. This was subsequently divided into two equal volumes of $15 \mathrm{~mL}$ to create two groups of controls and glucose incubations. To the glucose incubation group, glucose was added to create an overall sugar concentration of 0.5 M. Both groups were filter sterilised using syringe filters. All samples were statically incubated at $37^{\circ} \mathrm{C}$ for 30 days. After removal from incubation the glucose samples underwent buffer exchange to remove free glucose using Centripure P100 columns with $25 \mathrm{mM}$ MOPS, $115 \mathrm{mM} \mathrm{NaCl} \mathrm{pH} \mathrm{7.4.} \mathrm{The} \mathrm{solu-}$ tions were then concentrated to $12 \mathrm{mg} \mathrm{mL}^{-1}$ using Amicon Ultra $0.5 \mathrm{~mL}$ centrifugal filter units with a $10 \mathrm{kDa}$ cut off.

Glycation of lysozyme for quantification. Lysozyme was prepared as $5 \mathrm{mg} \mathrm{mL}^{-1}$ solution in $(200 \mathrm{~mL}) 25 \mathrm{mM}$ MOPS, $115 \mathrm{mM} \mathrm{NaCl}$, adjusted to $\mathrm{pH}$ 7.4. This was subsequently divided into 2 equal volumes of $100 \mathrm{~mL}$ to create two groups of controls and glucose incubations. To the glucose incubation group glucose was added to create an overall sugar concertation of $0.5 \mathrm{M}$. Both groups were filter sterilised using syringe filters. All samples were statically incubated at $37^{\circ} \mathrm{C}$ for 30 days. After removal from incubation the glucose samples underwent buffer exchange.

Time course mass spectrometry. Lysozyme glycation time course experiments were carried out on a Waters Synapt G2 (Waters, Manchester, UK) IM-MS instrument (Fig. S1 and Table $\mathrm{S} 1 \dagger$ ). Experiments were carried out in positive ionisation and resolution mode at collision energies of $4 \mathrm{~V}$ and $20 \mathrm{~V}$. The instrument parameters are summarised in the ESI (Time course mass spectrometry $\dagger$ ). Lysozyme samples were solubil- ised in acidified ammonium acetate solution (200 mM AmAc, $0.01 \%$ formic acid) and diluted to a concentration of $\sim 5 \mu \mathrm{M}$ protein whereby acidification was employed to improve signal. All subsequent data analysis software and procedures are outlined within the ESI. $\dagger$

Lysozyme quantification and albumin glycation mass spectrometry. $12 \mathrm{mg} \mathrm{mL}^{-1}$ glycated and non-glycated lysozyme in MOPS with $1 \mathrm{M}$ ammonium acetate solution $\mathrm{pH} 7.2$ were analysed at a final protein concentration of $\sim 4 \mu \mathrm{M} .12 \mathrm{mg} \mathrm{mL} \mathrm{m}^{-1}$ albumin in MOPS was buffer exchanged into $1 \mathrm{M}$ ammonium acetate solution $\mathrm{pH}$ 7.2. Optimal MS resolution was achieved for the glycated form of albumin by diluting the buffer exchanged sample at a 1:40 ratio into a water/formic acid solution (99.9: 0.1, v:v\%) at which the final protein concentration was $\sim 5 \mu \mathrm{M}$ prior to analysis. For consistency, the nonglycated form of albumin was also treated in the same manner. All experiments were performed using nano-electrospray ionisation (nESI) in positive ionisation mode. Experiments were performed on a Q Exactive UHMR hybrid quadrupole-orbitrap mass spectrometer (Thermo Fisher scientific). A full list of the Q Exactive settings utilised are outlined in Table S3. $\dagger$ Mass spectra were recorded at a resolving power of 200000 (lysozyme) and 3125 (albumin). Full quantification and cations are shown in the ESI $\dagger$ and within Fig. S3-S4 and Tables S4-S6. $\dagger$

FTIR-ATR. FTIR-ATR spectra were recorded on a Bruker Alpha FTIR spectrometer fitted with a platinum ATR module. Spectra were recorded from 4000 to $400 \mathrm{~cm}^{-1}, 24$ coadded scans with a resolution of $4 \mathrm{~cm}^{-1}$. All spectra were measured using lyophilised lysozyme that had been previously buffer exchanged to Millipore grade $\mathrm{H}_{2} \mathrm{O}$. The spectra were analysed in Matlab R2018a (The MathWorks, Natick, MA, USA) using an in-house tool box. All FTIR data were an average of 5 replicates. Raw and pre-processed spectra are shown in Fig. S2.†

Raman spectroscopy. Raman measurements were undertaken on a Renishaw inVia Raman microscope (Renishaw Plc., Gloucestershire, UK) using a $785 \mathrm{~nm}$ laser with a laser power on the sample of $\sim 30 \mathrm{~mW}$. The experimental parameters used for all data collection were $10 \mathrm{~s}$ exposure and 12 accumulations resulting in an overall acquisition time of $120 \mathrm{~s}$ per measurement. Samples were prepared as $12 \mathrm{mg} \mathrm{mL}^{-1}$ at $360 \mu \mathrm{L}$ in the concentration gradient summarised in Table S7† totaling 19 different samples. The set-up consisted of a 96 well quartz plate (Hellma ${ }^{\mathrm{TM}}$ ) using randomised samples with three repeats per well. Raw and pre-processed spectra are shown in Fig. S5. $\dagger$

Data pre-processing and chemometrics. All data pre-processing and subsequent analysis was performed using Matlab R2018a with in house toolboxes (these are available via https:// github.com/Biospec/). For FTIR-ATR the data were baselined and normalised to allow comparison. The Raman spectra were baselined, normalised and smoothed (full pre-processing methods are described in ESI $\dagger$ ). The data set was then divided into two sets in order to generate a PLSR linear predictive model. The data set included 19 concentrations of glycated lysozyme mixed with control lysozyme creating a $\sim 6 \%$ gradient from 0 to $100 \%$ glycated samples. This produced 19 datasets 
of which three were removed due to being outliers (Fig. S6†). The training set and test set therefore included 8 datasets each with three spectral repeats per concentration. The optimum number of latent variables (PLS factors) was chosen as lowest latent variable number with the lowest root-mean-squared error on the cross-validation set (RMSEcv). In this case it was 3 ; i.e., the least number of PLS factors needed to decrease the RMSEcv. Leave-one-out cross validation was used to test each model. The chosen model with $\mathrm{LV}=3$ was used to run the test set and generate an $R^{2}$ and $Q^{2}$ for the model.

\section{Results and discussion}

Lysozyme has 6 Lys residues and an N-terminus that can be glycated by glucose via a condensation reaction (Fig. 1). The project used two experimental groups. The first group was $5 \mathrm{mg} \mathrm{mL} \mathrm{m}^{-1}$ lysozyme with the addition of $0.5 \mathrm{M}$ glucose in incubation buffer. The control group was $5 \mathrm{mg} \mathrm{mL}^{-1}$ lysozyme in incubation buffer without glucose. Each of the conditions had 7 vials that were incubated at $37{ }^{\circ} \mathrm{C}$. One vial from each condition was collected at day 1, 2, 5, 7, 14, 21 and day 30 to study the structure of lysozyme as glycation increases. The glycation method was adapted from previous literature. ${ }^{6}$ The time length of 30 days was chosen to maximise the amount of glycation in an incubation time frame that avoided the use of preservatives such as sodium azide to prevent bacterial growth.

\section{Mass spectrometry determination of glycation}

To determine the extent of glycation, MS was employed to determine the mass changes in the protein as a function of incubation time. The MS analysis was carried out using the parameters stated in the methods section whilst the results are shown in Fig. S1.† Table 1 summarises the results of the glucose incubation and monitors its covalent binding to lysozyme with incubation time. In order to check that glucose was covalently bound, the mass spectrometry analysis was carried out at two different collisional energies of $4 \mathrm{~V}$ and $20 \mathrm{~V}$ with the assumption that at $20 \mathrm{~V}$ only covalently bound glucose should be seen and any that is non-covalently associating to the protein would be removed. At both voltages, the populations (\%) of glycated species were similar, as shown from the SE in Table 1, showing that no glucose was bound non-co- valently. The table summarises the integrated mass peaks averaged over $20 \mathrm{~V}$ and $4 \mathrm{~V}$, using the $8^{+}$and $7^{+}$charge states for both voltages. The full quantitative analysis is described in the ESI (Time course mass spectrometry $\dagger$ ). The protein is $100 \%$ non-glycated at day 0 . Lysozyme becomes increasingly glycated the longer it is incubated with glucose and thus the glycated population increases with time. After 30 days the most populated state of lysozyme is that with 1 or 2 bound glucose molecules. In this analysis, as far as we can tell, we never saw more than 4 Lys glycated or any significant change in protein glycation status after 21 days, suggesting that 3 of the Lys side chains are not susceptible to being glycated. The difference in susceptibility to being glycated is a result that has been widely published and investigated using peptide mapping. It is thought to arise from the surrounding environment and solvent accessibility with Lys residues less open to the solvent or having amine groups hydrogen bonded to other protein groups being less likely to be glycated. ${ }^{22,30,31}$

\section{FTIR-ATR analysis of glycated lysozyme}

FTIR-ATR was carried out on all the controls and glycated lysozyme from days 1 to 30 of the incubation using lyophilised samples outlined in the methods. The full FTIR spectra of all of the samples are shown in Fig. 2a, where the amide region of the spectrum stretches from $1700 \mathrm{~cm}^{-1}$ to $1200 \mathrm{~cm}^{-1}$ and contains amide regions I, II and III. The overlap between sugar peaks and amide peaks becomes increasingly complicated as increasing sugar peaks can stretch from $1500 \mathrm{~cm}^{-1}$ to $1000 \mathrm{~cm}^{-1} \cdot{ }^{32}$

Amide $\mathrm{I}$ is represented by the peak at $1649 \mathrm{~cm}^{-1}$ and amide II at $1537 \mathrm{~cm}^{-1}$. Amide III is $1452 \mathrm{~cm}^{-1}$ and $1390 \mathrm{~cm}^{-1}$ with some possible overlap with the sugar peaks. The predominant changes are in at the sugar region around $1000 \mathrm{~cm}^{-1}$ $1200 \mathrm{~cm}^{-1}$ and are highlighted in Fig. 2a where a unique peak in the spectra of the glycated samples can be seen at $1030 \mathrm{~cm}^{-1}$ (highlighted in purple) which is not present in the control samples. A full spectral assignment is given in Table S2. $\dagger$

In order to determine the specific peak changes, PCA was carried out on the glycated samples. PCA scores plots are used to highlight patterns within a dataset, in this case spectral variation between the peaks from the samples. It can be seen from

Table 1 Summary of glycation \% calculated from the integration of the $7^{+}$and $8^{+}$charge states (most intense), at $4 \mathrm{~V}$ and $20 \mathrm{~V}$ collisional energies of lysozyme using peaks that increase by $162 \mathrm{Da}$ (addition of glucose $-\mathrm{H}_{2} \mathrm{O}$ ). The \% of glycation was monitored over time course from day 0 to day 30. Errors were calculated as the standard error (SE) of 4 peak integrations from each sample $(4 \mathrm{~V}$ and $20 \mathrm{~V}$ including both the $7+$ and $8+$ charge states. Analysis carried out using. Waters Synapt G2 (Waters, Manchester UK). Calculations and masses are shown in Table S1 $\dagger$

Population \% of lysozyme with bound glucose as a function of time

\begin{tabular}{|c|c|c|c|c|c|c|c|c|}
\hline Number of glucose bound & Day 0 (Lys standard) & Day 1 & Day 2 & Day 5 & Day 7 & Day 14 & Day 21 & Day 30 \\
\hline 0 Glucose & $100 \pm 0$ & $61 \pm 4$ & $58 \pm 3$ & $46 \pm 2$ & $40 \pm 3$ & $19 \pm 1$ & $13 \pm 1$ & $12 \pm 1$ \\
\hline 1 Glucose & 0 & $27 \pm 2$ & $27 \pm 1$ & $41 \pm 0$ & $41 \pm 1$ & $40 \pm 1$ & $35 \pm 2$ & $36 \pm 1$ \\
\hline 2 Glucose & 0 & $13 \pm 2$ & $11 \pm 1$ & $18 \pm 1$ & $18 \pm 2$ & $30 \pm 1$ & $33 \pm 1$ & $35 \pm 1$ \\
\hline 3 Glucose & 0 & 0 & $4 \pm 1$ & $5 \pm 1$ & $5 \pm 1$ & $11 \pm 1$ & $14 \pm 2$ & $14 \pm 1$ \\
\hline 4 Glucose & 0 & 0 & 0 & 0 & 0 & 0 & $5 \pm 1$ & $4 \pm 0$ \\
\hline
\end{tabular}




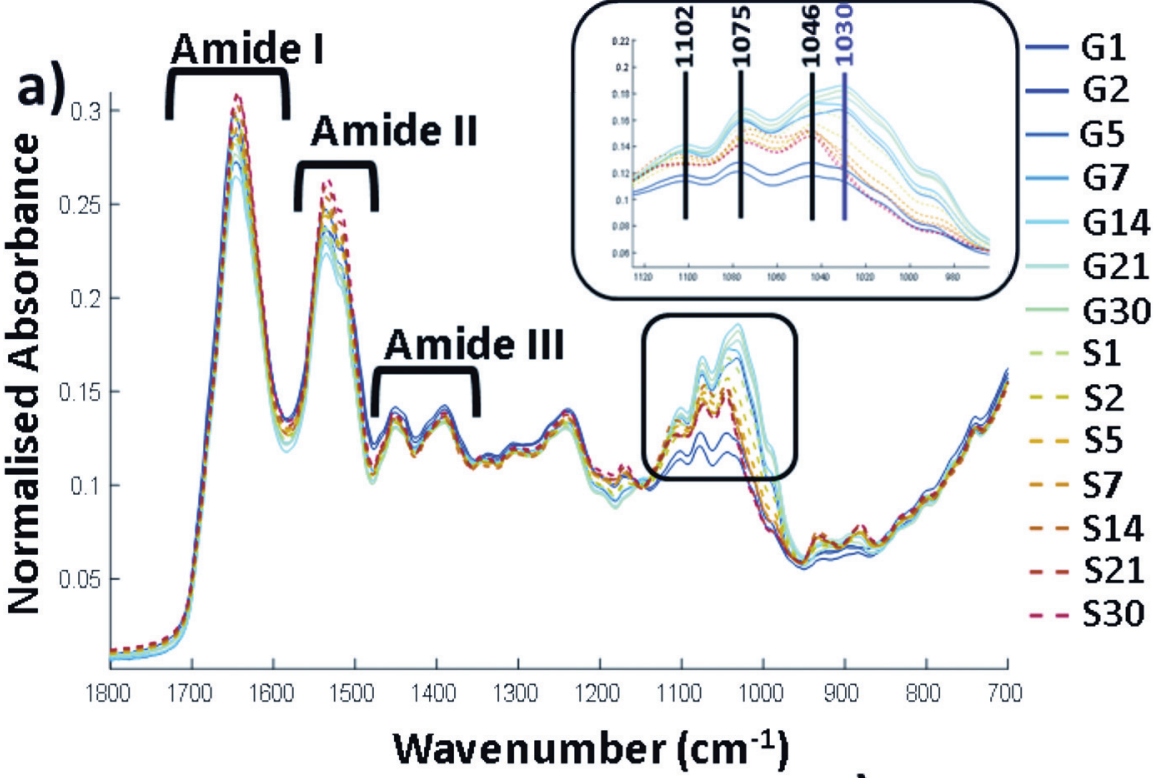

Amino Acids / Sugars

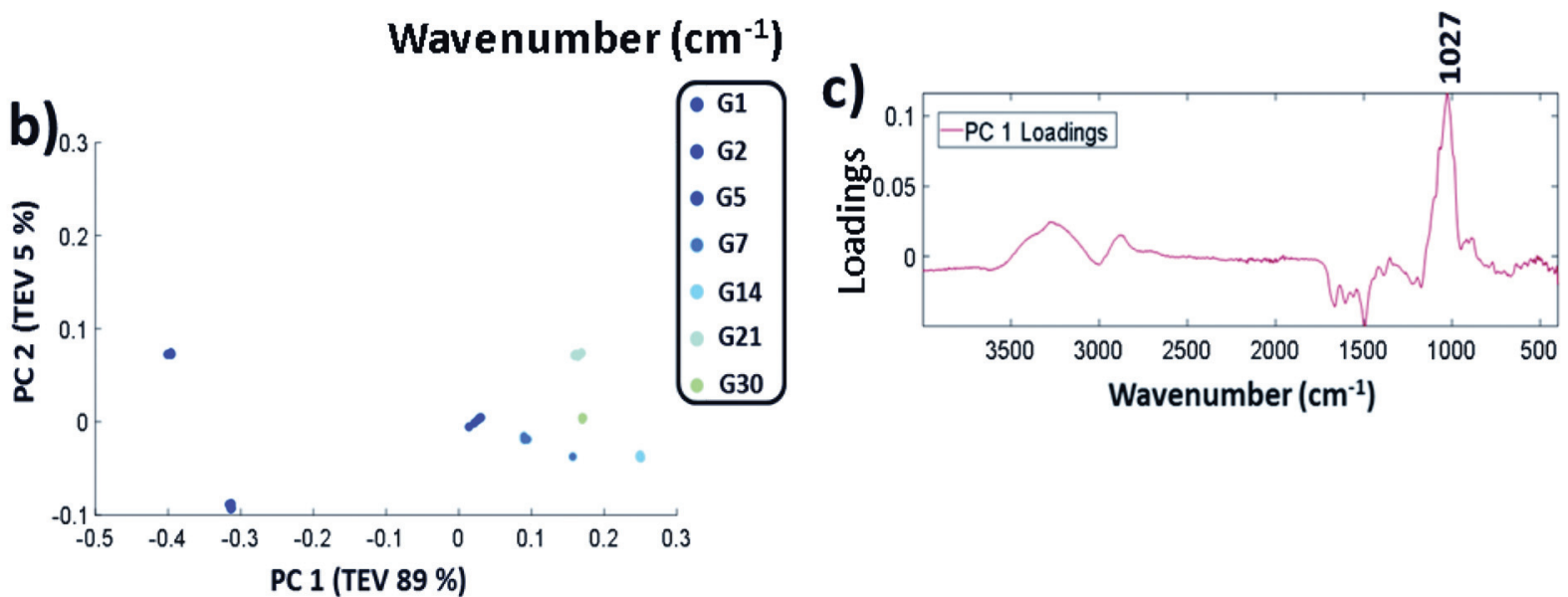

Fig. 2 (a) FTIR-ATR spectrum showing the controls (samples incubated with no glucose labelled S and glycated lysozyme G from incubation day 1 through until day 30. Spectra are cut to only show between $1800 \mathrm{~cm}^{-1}$ to $700 \mathrm{~cm}^{-1}$, b) PCA scores plot carried out across full spectra $\left(400-4000 \mathrm{~cm}^{-1}\right)$ and (c) the corresponding PCA loadings plot. Samples were filtered, buffer exchanged to $\mathrm{H}_{2} \mathrm{O}$ and stored at $-20{ }^{\circ} \mathrm{C}$ until the analysis was undertaken. The FTIR was carried on solid samples at $25^{\circ} \mathrm{C}$ using the parameters stated in the methods. All FTIR data is an average of 5 replicates per sample. The data were scaled to allow a more accurate comparison between the samples as described in ESI (Data pre-processing and chemometrics $\dagger$ ). TEV = total explained variance.

Fig. 2b that the samples separated out on PC1 that accounts for $89 \%$ of the total explained variance (TEV) with a general trend from G1 to G30. The corresponding loadings plot (Fig. 2c) can be used to highlight the most important peaks in terms of identifying the variability between samples. A peak at $1027 \mathrm{~cm}^{-1}$ has been identified as the main peak causing the separation across PC1. We therefore deduce that the unique peak in the FTIR-ATR spectra in Fig. 2a at 1030/1027 $\mathrm{cm}^{-1}$, due to $\mathrm{C}-\mathrm{O}$ or $\mathrm{C}-\mathrm{N}$ bonds, is caused by glycation of lysozyme.

Fig. 3 shows three FTIR-ATR spectra to determine the difference between free glucose and bound glucose peaks. Lysozyme mixed with free glucose in solution and then freeze dried has a main peak at $1022 \mathrm{~cm}^{-1}$. The $\mathrm{S} 30$ sample of lysozyme incubated with no glucose for 30 days has a peak at $1046 \mathrm{~cm}^{-1}$ therefore further validating that in $\mathrm{G} 30$ the peak at $1030 \mathrm{~cm}^{-1}$ is only found in glycated samples and could be attributed to covalently bound glucose only.

\section{Analysis of glycation using Raman spectroscopy}

Raman spectroscopy is a complementary vibrational analysis technique to FTIR spectroscopy with the benefit of having little spectral contribution from water. A larger scale glycation set-up was used in order to create two sets of samples: lysozyme was incubated for 30 days, with and without glucose. Raman spectroscopy requires a minimum concentration of $>10 \mathrm{mg} \mathrm{mL}^{-1}$ and the 96 well plate set-up needs $360 \mu \mathrm{L}$ per well. We therefore simplified the incubation set-up in order to create larger volumes so we could create a concentration gradient. Glycated samples were buffer exchanged to remove free glucose (full details are described in sample preparation). The 


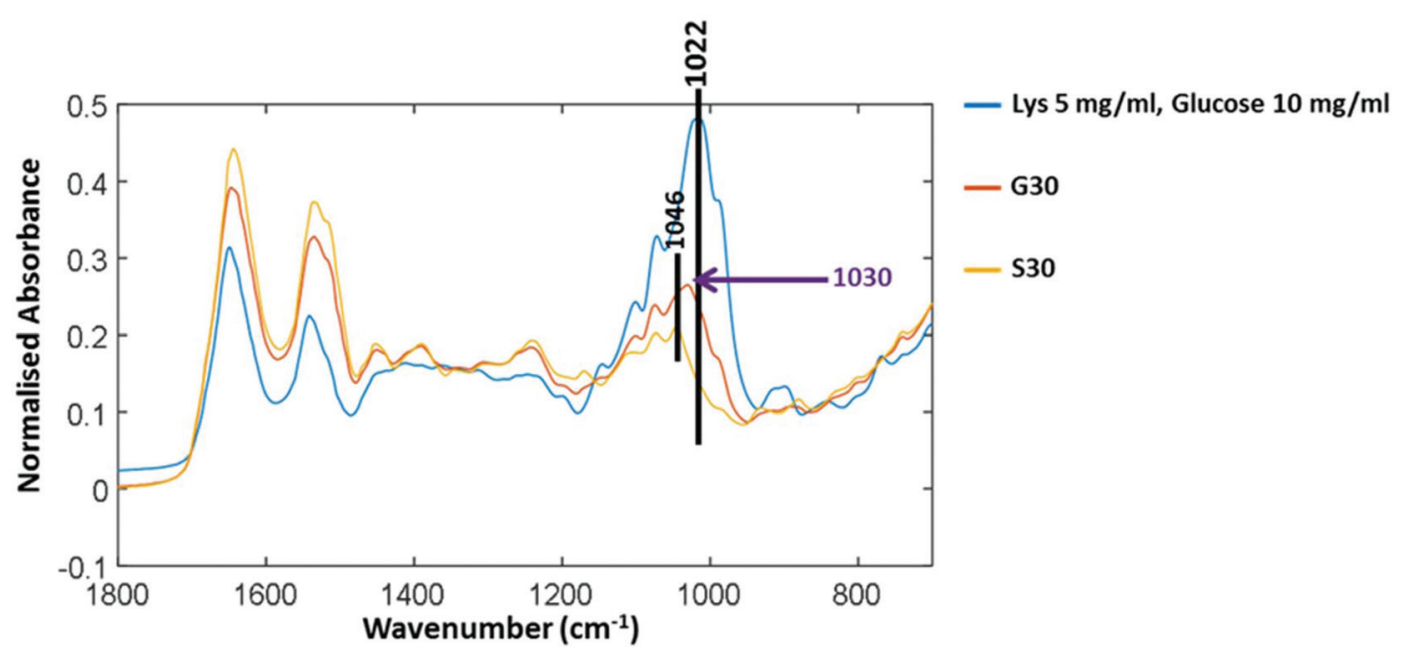

Fig. 3 FTIR-ATR spectra of control lysozyme (S30) after 30 days of incubation with no glucose; glycated lysozyme after 30 day (G30); and lysozyme mixed with free glucose. Samples are lyophilized and analysed under the same conditions to compare bound and free glucose peaks.

Table 2 Summary of glycation \% of lysozyme after 30 days incubation at $5 \mathrm{mg} \mathrm{mL}^{-1}$ with $0.5 \mathrm{M}$ glucose. Analysis carried out using $Q$ Exactive UHMR hybrid quadrupole-orbitrap mass spectrometer (Thermo Fisher scientific). Integrations were carried under the $7^{+}$charge state (only one intense enough to analyse). Full calculations and masses are shown in Fig. S3 and Table $\mathrm{S} 4 \dagger$

\begin{tabular}{lll}
\hline $\begin{array}{l}\text { Population of } \\
\text { lysozyme with } \\
\text { bound glucose }\end{array}$ & $\begin{array}{l}\text { Control (\%) } \\
\text { 30 day incubation } \\
\text { (no glucose) }\end{array}$ & $\begin{array}{l}\text { Glycated (\%) } \\
\text { 30 day glucose } \\
\text { incubation }\end{array}$ \\
\hline 0 & 100 & 24 \\
1 & 0 & 47 \\
2 & 0 & 23 \\
3 & 0 & 6
\end{tabular}

samples were anlaysed using MS, which is summarised in Table 2 below. In comparison to the first glycation (Table 1) this method shows that although the most abundant species is lysozyme with 2 glucose bound, there are fewer lysozymes with 3 glucose bound, suggesting that the altered reaction conditions may affect the glycation rate.

The samples were concentrated to $12 \mathrm{mg} \mathrm{mL}^{-1}$ and a concentration gradient was created by spiking glycated lysozyme into the control samples to create a series of $0 \%$ to $100 \%$ glycated lysozyme samples. The full concentration gradient is shown in Table S7. $\dagger$ Using a 96 well plate set-up, described in the Experimental section, the samples were run in a randomised order so as to avoid bias from laser power variation between the wells, or for any time-dependent changes. The samples were measured in triplicate and the average of each sample is shown in Fig. 4a. The $39 \%, 78 \%$ and $94 \%$ glycated samples were outliers and were removed (PCA including these data points are shown in Fig. S6†).

The full Raman spectra are shown in Fig. 4a. The vibrational frequency regions can be split up similarly to that of FTIR-ATR where $1600-1800 \mathrm{~cm}^{-1}$ is the Amide I,
$1400-1500 \mathrm{~cm}^{-1}$ is the Amide II and $1200-1400 \mathrm{~cm}^{-1}$ is the Amide III region. At lower wavenumbers, such as between 750-1100 $\mathrm{cm}^{-1}$, the amino acid and sugar molecules vibrational frequencies result from $\mathrm{C}-\mathrm{O}$ bonds vibrations and others. A full spectral assignment can be found in Table S8. $\dagger$ In general, it is difficult to pull out spectral differences between the \% glycation of samples in Fig. 4a. Therefore, in order to highlight smaller spectral differences and to look at correlations within the data, we have used PCA with the three spectral repeats for each sample plotted separately. PC $1 v s$. PC 2 showed no clear separation between glycated and non-glycated samples; by contrast, PC 1 plotted against PC 3 (Fig. 4b) separated the spectra on amount of lysozyme glycation. The three repeats from each sample show good reproducibility as they can be seen to cluster together for each set. Fig. 4c shows the corresponding loadings plot. PC 1 loadings are difficult to interpret but separation does depend on a peak at $1452 \mathrm{~cm}^{-1}$ which is the $\mathrm{CH} / \mathrm{CH}_{2} / \mathrm{CH}_{3}$ vibrational frequency. The peaks in PC 3 were found to correspond to glucose peaks. PC 3 loadings highlight important spectral bands within the sugar regions at $1038 \mathrm{~cm}^{-1}, 1060 \mathrm{~cm}^{-1}$ and $1121 \mathrm{~cm}^{-1}$, reflecting the level of glycation of lysozyme.

In order to help distinguish glycated protein from protein with free glucose we again performed a glucose spike in experiment with lysozyme (Fig. 5). Furthermore, we glycated albumin, a protein that is known to be glycated in the blood of diabetic patients. ${ }^{33}$ Human albumin has an expected mass of $\sim 66.5 \mathrm{kDa}$, congruent with our MS findings (shown in Table S6 $\dagger$ ). Albumin has 59 Lys, $23 \mathrm{Arg}$ and the N-terminal amine that can potentially be glycated. Lys side chains are the most susceptible to be glycated. ${ }^{34}$ Peptide mapping is often used to quantify and identify the glycation sites with the number of glycation sites dependent upon the method of glycation used. Albumin is usually reported to have higher levels of glycation than lysozyme. Bovine serum albumin incubated with glucose in a similar manner showed that up to 48 glucose 


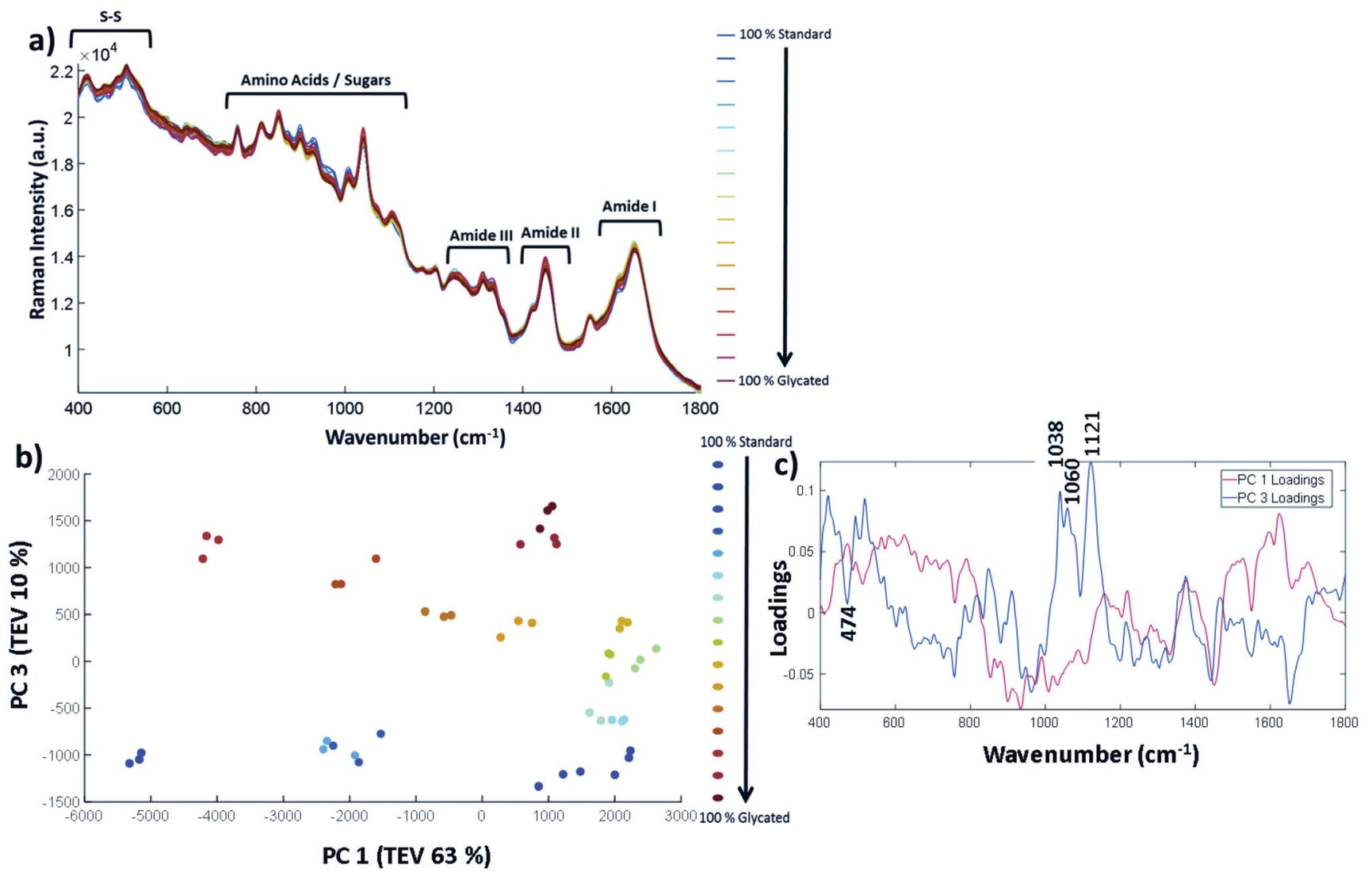

Fig. 4 Raman spectra and PCA of glycation concentration with 9\%, 78\% and 94\% glycated sample outliers removed. (a) Pre-processed Raman spectra with three repeats per sample, (b) PCA scores plot of samples excluding outliers showing PC 1 has a total explained variance (TEV) of $63 \%$ and PC 3 has a TEV of $10 \%$, and (c) the corresponding loadings plot to highlight peaks that contributed to the separation of samples in the PCA.

molecules had bound to the protein. ${ }^{35}$ Increasing the temperature can push this number up to $\sim 57 .^{36}$ Quantification of human serum albumin (HSA) from diabetic patients has found up to 15 Lys residues with glycation. ${ }^{37}$ Therefore albumin serves as model protein that is able to achieve higher levels of glycation than that of lysozyme. Glycation levels of albumin are dependent on the conditions used to induce glucose binding. From our MS analysis we can estimate that 9-24 glucose molecules bound to albumin with the most prevalent number of glycations being 17 under our experimental conditions (Fig. S4 \& Tables S5-S6†).

Glycated and non-glycated albumin were produced using identical methods to that of lysozyme detailed in the Experimental section. The inclusion of a further protein that can become glycated and is of medical importance was used to determine whether the vibrational bands identified originated from bound glucose and could be translated to other proteins.

The spectra in Fig. 5 are plotted without buffer subtraction due to the addition of spiked in glucose changing the baseline slope and intensity. The spectra have been normalised and baselined using EMSC to allow comparison. Fig. 5a shows the three repeated spectra of lysozyme control, lysozyme glycated and lysozyme with glucose spike. We have also included a spectrum of glucose in the same buffer ( $25 \mathrm{mM}$ MOPS and
$115 \mathrm{mM} \mathrm{NaCl}$ at $\mathrm{pH} 7.4$ ) in Fig. $\mathrm{S} 8 \dagger$ for comparison. The labelled peaks are ones which showed a spectral shift between the glycated samples and the spiked in glucose at peak positions already attributed to different forms of glucose. In the sample with spiked in glucose the intensity of the peak at $1121 \mathrm{~cm}^{-1}$ arising from the $\mathrm{C}-\mathrm{OH}$ vibration increases. This peak is very weak and broad in both the control and glycated lysozyme. In the $\mathrm{CH}_{2} / \mathrm{CH}_{3}$ region there is a peak shift from $1448 \mathrm{~cm}^{-1}$ in the spiked glucose sample to $1452 \mathrm{~cm}^{-1}$ in the glycated and control sample, suggesting that vibrations arising from $\mathrm{C}-\mathrm{H}$ stretching are different in glucose than in the protein. The Amide I region also shows a slight shift in the band at $1650 \mathrm{~cm}^{-1}$ in the control and glycated protein to $1642 \mathrm{~cm}^{-1}$ in the spiked sample suggesting a change in the $\alpha$-helical content of lysozyme, but this could be obscured by the $\mathrm{C}=\mathrm{O}$ of the aldehyde group in the open form of glucose. Similar spectral differences can be picked out in Fig. $5 \mathrm{~d}$ for albumin, where $1121 \mathrm{~cm}^{-1}$ is the main peak shown in the glucose spike and subtle spectral shifts again are seen for $\mathrm{CH}_{2} /$ $\mathrm{CH}_{3}$ and the amide I region. We again used PCA to pull out smaller differences in the spectra for both lysozyme and albumin. Fig. 5b and e show the three spectral repeats of each sample. In both PCA plots, PC1 accounts for by far the largest total explained variance. In general the PCA plots are very 

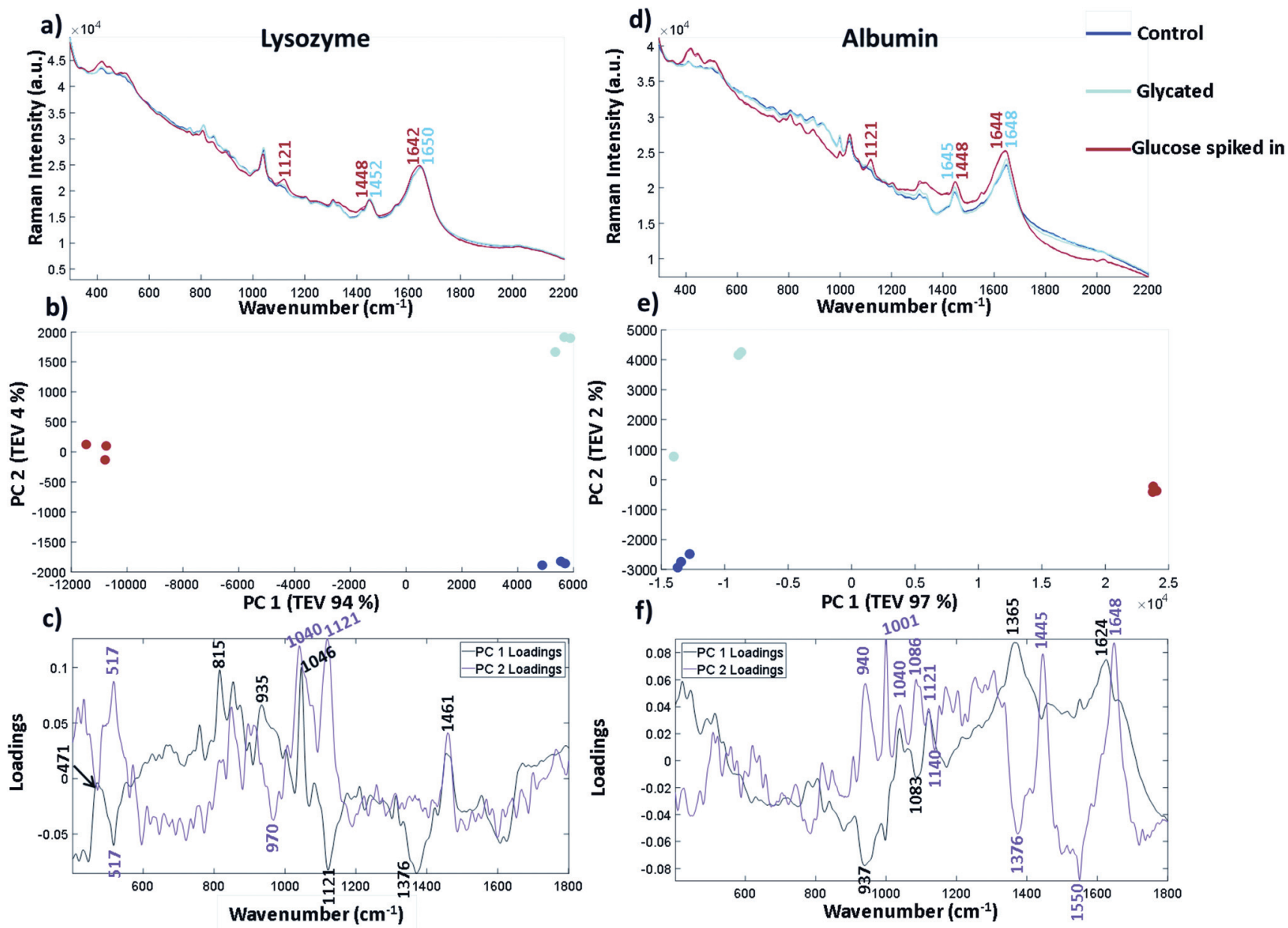

Fig. 5 Raman spectra of lysozyme and albumin all shown as an average of three replicates. (a) and (d) show lysozyme and albumin at $12 \mathrm{mg} \mathrm{mL}^{-1}$ in $25 \mathrm{mM}$ MOPS and $115 \mathrm{mM} \mathrm{NaCl}$ at pH 7.4 glycated, control and with $10 \mathrm{mg} \mathrm{mL}^{-1}$ glucose added. (b) and (e) PCA scores plots of the albumin and lysozyme samples showing the three separate replicates per condition. (c) and (e) show the corresponding PCA loadings plot for lysozyme and albumin. Both loadings plots highlight peaks at $1040 \mathrm{~cm}^{-1}$ as being significant to the separation in PC 2 .

similar, showing a mirror image of one another along the $y$-axis suggesting the spectral differences in the samples follow a similar pattern of separation. For lysozyme, the most significant peaks that led to the separation of the data points in lysozyme across PC 1 are $1046 \mathrm{~cm}^{-1}, 1121 \mathrm{~cm}^{-1}$ and $1376 \mathrm{~cm}^{-1}$ shown from the intensity of the corresponding loadings plot in Fig. $5 \mathrm{c}$ where $1121 \mathrm{~cm}^{-1}$ and $1376 \mathrm{~cm}^{-1}$ are more intense in the spiked sample. The peak at $1046 \mathrm{~cm}^{-1}$, which is increased in the glycated and control, is assigned to $\mathrm{C}-\mathrm{O}$ vibration of lysozyme in the Raman spectrum summarised in Table S8. $\dagger$ It can also be seen in FTIR-ATR (Fig. 3). Therefore, PC 1 separates spectra based on the free glucose in solution from the glucose spiked in sample. PC 2, on the $y$-axis, accounts for only $4 \%$ of the variance for lysozyme, but still accurately separates the samples. The PC 2 loadings in Fig. $5 \mathrm{c}$ highlight peaks at $1040 \mathrm{~cm}^{-1}$ and $1121 \mathrm{~cm}^{-1}$ as contributing to the most variance. Both these bands are increased in the glycated sample, but not in control samples. Thus $1040 \mathrm{~cm}^{-1}$ and $1121 \mathrm{~cm}^{-1}$ appear to distinguish between glycated and control samples with or without buffer subtraction.
In albumin, PC1 is dominated by an increase in peaks at $1624 \mathrm{~cm}^{-1}$ and $1365 \mathrm{~cm}^{-1}$ in the glycated samples (Fig. 5f). The peak at $1624 \mathrm{~cm}^{-1}$ is usually assigned to changes of protein structure and can indicate increased $\beta$-sheet formation. Alternatively it may report on Tyr/Trp/Phe, where an increase in intensity would suggest unfolding. Ultimately these results suggest that incubation of lysozyme at high concentration may be destabilising the protein; this seems to be reversible as Fig. 5d shows no secondary structure change after free glucose removal from glycated albumin. ${ }^{38}$ The peak at $1365 \mathrm{~cm}^{-1}$ is assigned to the $\mathrm{CH}_{2}$ groups of free glucose. PC 1 also shows that there is loss of a peak at $937 \mathrm{~cm}^{-1}$ in the glucose spiked samples assigned to $\mathrm{N}-\mathrm{C}-\mathrm{C}$ which is usually vibrations from the protein backbone. However, a peak at $940 \mathrm{~cm}^{-1}$ is also dominant in PC 2 in the glycated samples and therefore it could be $\mathrm{N}-\mathrm{C}-\mathrm{C}$ vibrations from the formation of Amadori products. PC 2 also highlights peaks at $1040 \mathrm{~cm}^{-1}$ and $1083 \mathrm{~cm}^{-1}$ in the sugar region. The peak at $1040 \mathrm{~cm}^{-1}$, similarly to lysozyme, shows an increase in intensity, whereas the peak at $1086 \mathrm{~cm}^{-1}$ is only present in the glycated samples 
in PC 2. These peaks are not assigned to free glucose in the same buffer (Fig. S8 $\dagger$ ). Overall the peak at $1027 / 1030 \mathrm{~cm}^{-1}$ in FTIR-ATR for glycated lysozyme and the peaks at 1040/ $1038 \mathrm{~cm}^{-1}$ and $1121 \mathrm{~cm}^{-1}$ in Raman for both lysozyme and albumin seem to be increasing in intensity in glycated only samples. Albumin shows a further peak at $1086 \mathrm{~cm}^{-1}$ that is only present in glycated samples. These peaks all originate from the $\mathrm{C}-\mathrm{O}$ vibrations and are within the sugar region of a Raman spectrum.

\section{Quantification of glycation}

In order to determine the ability of Raman to quantify the extent of glycation in lysozyme, we used PLSR to generate a linear predictive model. To produce samples for liquid Raman analysis, a larger incubation volume was needed in which lysozyme was glycated using the same conditions as before, but in two large vials of $100 \mathrm{~mL}$ each for control (without glucose) and glycated (with $0.5 \mathrm{M}$ glucose). The samples were again filter buffer exchanged into $25 \mathrm{mM}$ MOPS, $115 \mathrm{mM} \mathrm{NaCl}$ at $\mathrm{pH}$ 7.4 and were concentrated to $12 \mathrm{mg} \mathrm{mL}{ }^{-1}$. From these samples, a concentration gradient was created (Table S7 $^{\text {) }}$, where we had concentrations of glycated mixed with control samples to create a total volume of $360 \mu \mathrm{L}$ and a concentration gradient of $0 \%$ to $100 \%$ glycated in increments of $\sim 6 \%$. As the lysozyme could have anywhere between 0-3 lysine residues glycated, the known \% glycation was an approximation. This generated a total of 19 samples, three of which were removed due to being outliers, leaving a total of 16 samples, 8 of which were used for a training set and 8 for the test set.

The results for PLSR model are shown in Fig. 6 and the statistics summarised in Table 3 based on the 3 PLS factors using leave-one-out cross validation. The model chosen uses the minimum number of PLS factors needed in the leave-one-out cross validation to obtain a low root mean square error of cross validation (RMSEcv), which in this case was 3. Here the $R^{2}$ value provides a measure of how well a linear model fits the training data and the $Q^{2}$ is the same linear correlation coeffi-

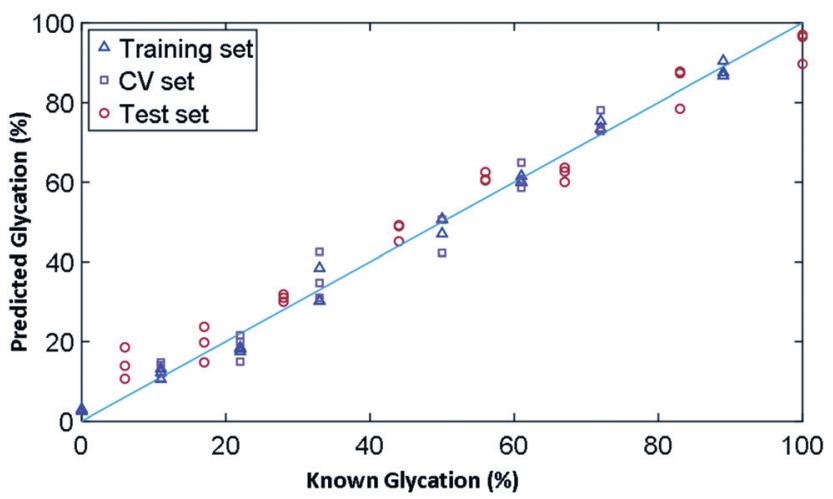

Fig. 6 PLSR linear predictive model of \% glycation of lysozyme from 0 to $100 \%$ in $\sim 6 \%$ increments totaling 16 concentrations. The model was built on 8 data sets each with three spectral repeats. The model was tested on the remaining 8 data sets. The model test set had a $Q^{2} p$ of 0.97 and an RMSEp of $5.53 \%$.
Table 3 Summary of the statistical analysis of the PLSR predictive model

\begin{tabular}{lll}
\hline & PLSR statistics & Value \\
\hline Training set & $R^{2}$ & 0.99 \\
Cross-validation set & RMSEc & 2.80 \\
& $Q^{2}$ cv & 0.96 \\
Test set & RMSEcv & 5.31 \\
& $Q^{2} \mathrm{p}$ & 0.97 \\
& RMSEp & 5.53
\end{tabular}

cient for the two types of model. $Q^{2} \mathrm{cv}$ is the performance on prediction based on leave-one-out cross validation where as $Q^{2} \mathrm{p}$ is the performance prediction of the model on the test data set; these are the 8 samples left out of the model and not used during cross validation. A robust model seeks to have $Q^{2}$ values close to the $R^{2}$, with a perfect linear relationship being 1. Overall, the training set fits the model with an $R^{2}$ value of 0.99 and a root mean square error of covariance (RMSEc) of $2.80 \%$. The model, with 3 PLS factors, had a $Q^{2} \mathrm{cv}$ of 0.96 and $Q^{2}$ p of 0.97 . The root mean squared error for cross validation for predictive ability of the model (RMSEp) is $5.53 \%$, showing that we had a very robust model for the prediction of glycation $\%$ from spectroscopic data.

\section{Conclusion}

Vibrational spectroscopy has successfully been used to distinguish between glycated and non-glycated proteins. For the first time, we have demonstrated the sensitivity of Raman spectroscopy to quantify glycation of proteins in solution. Furthermore, we used a 96 well plate set-up suggesting the potential for high throughput, automated analysis of protein samples. Raman spectroscopy analysis of glycated proteins in less than two minutes allows rapid screening thus providing proof-of-concept for the development of Raman spectroscopy for in-line protein characterisation.

Lysozyme was incubated with glucose over a 30 day period and samples were removed at regular intervals for a time course analysis. Mass spectrometry was used to determine the number of bound glucose molecules to the protein. Lysozyme was glycated to some extent within 24 hours. By day 30, the most abundant species was lysozyme with one glucose molecule bound though up to 4 was possible. Lyophilised samples from each time course interval were anlaysed using FTIR-ATR. PCA identified a peak at $1027 \mathrm{~cm}^{-1}$ which correlated with glycation levels. This peak was not present in the control lysozyme or the lysozyme sample spiked with glucose and therefore we assign $1027 \mathrm{~cm}^{-1}$ to bound glucose.

Raman spectroscopy analysis required a larger incubation set-up using larger sample volumes required for solution analysis. These were generated using lysozyme incubated with glucose and a non-glycated control incubated under the same environmental conditions with no glucose. Both were incubated for 30 days. MS analysis revealed that after 30 days the 
main species present was again lysozyme with one glucose bound $(47 \%)$ up to a maximum of three (6\%). It can be noted here that the populations of glycated species in large set-ups differ to those in the small scale set-up for FTIR (36\% one glucose bound, $14 \%$ for three glucose bound) and therefore incubation factors such as volume may affect the glycation rate. These samples were buffer exchanged and concentrated to $12 \mathrm{mg} \mathrm{mL}^{-1}$ and then used to create 16 samples from 0 to $100 \%$ glycated at $\sim 6 \%$ intervals. Using a 96 well plate set-up, the samples were analysed with Raman spectroscopy via automation on a Raman microscope. PCA revealed a more complex variance, with more contributing peaks, across PC 1 and PC 2 compared to FTIR-ATR samples, due to the addition of vibrations of buffer and water from using samples in solution. Both the Raman spectra in Fig. 5a and loadings plots in Fig. 5c highlighted increased intensity of peaks at $1038 \mathrm{~cm}^{-1}$, $1060 \mathrm{~cm}^{-1}$ and $1121 \mathrm{~cm}^{-1}$ compared to that of the peaks in the control. Furthermore, the peaks highlighted at $1038 \mathrm{~cm}^{-1}$ and $1060 \mathrm{~cm}^{-1}$ were not found in free glucose alone (Fig. S8 $\dagger$ ). To confirm the relevance of these peaks to other glycated proteins, albumin was glycated using the same conditions. In Fig. 5 we compared both glycated albumin and lysozyme with controls and glucose spiked in to the control protein. The spectra and PCA loadings again highlighted peak intensities at $1040 \mathrm{~cm}^{-1}$ and $1121 \mathrm{~cm}^{-1}$ as important to distinguish between glycated and non-glycated control protein. Albumin, which was found to be most abundant with 17 glucose bound, had a peak at $1086 \mathrm{~cm}^{-1}$ which was only present in the glycated samples and therefore could indicate bound glucose only. The sensitivity of Raman spectroscopy to quantify and predict glycation was investigated using multivariate linear regression model. The concentration gradient was used to build a PLSR model which produced an accurate predictive model with an RMSEp of $5.53 \%$. Raman spectroscopy, when used with chemometrics, therefore has the capability to rapidly quantify protein glycation.

Overall, these studies show the scope of Raman spectroscopy in the identification and quantification of glycation in proteins central to disease diagnostics and quality control of protein therapeutics. The development of Raman spectroscopy in these fields would allow for rapid sample analysis in solution and therefore provide the foundation for evolving on-line and in-line analytical and diagnostic tools.

\section{Conflicts of interest}

There are no conflicts to declare.

\section{Acknowledgements}

This work was funded by a BBSRC CASE award with UCB Pharma BB/L014734/1. RG thanks the UK BBSRC (BB/L014823/ 1) for support for Raman spectroscopy.

\section{References}

1 L. C. Maillard, C. R. Hebd. Seances Acad. Sci., 1912, 154, 66-68.

2 J. S. Ramalho, C. Marques, P. C. Pereira and M. C. Mota, Eur. J. Ophthalmol., 1996, 6, 155-161.

3 C. Iannuzzi, G. Irace and I. Sirangelo, Front. Mol. Biosci., 2014, 1, 9.

4 J. M. Ashraf, S. Ahmad, G. Rabbani, A. Tasleem Jan, E. Ju Lee, R. Hasan Khan and I. Choi, IUBMB Life, 2014, 66, 686693.

5 M. Luthra and D. Balasubramanians, J. Biol. Chem., 1993, 268, 18119-18127.

6 S. Ghosh, N. K. Pandey, A. Singha Roy, D. R. Tripathy, A. K. Dinda and S. Dasgupta, PLoS One, 2013, 8, e74336.

7 J. M. Berg, J. L. Tymoczko, L. Stryer and L. Stryer, Biochemistry, W.H. Freeman, New York, 5th edn, 2002.

8 Q. Zhang, J. M. Ames, R. D. Smith, J. W. Baynes and T. O. Metz, J. Proteome Res., 2009, 8, 754-769.

9 A. Goldin, J. A. Beckman, A. M. Schmidt and M. A. Creager, Circulation, 2006, 114, 597-605.

10 V. P. Singh, A. Bali, N. Singh and A. S. Jaggi, Korean J. Physiol. Pharmacol., 2014, 18, 1-14.

11 A. Rahmadi, N. Steiner and G. Mü, Clin. Chem. Lab. Med., 2011, 49, 385-391.

12 C. Quan, E. Alcala, I. Petkovska, D. Matthews, E. Canova-Davis, R. Taticek and D. Ma, Anal. Biochem., 2008, 373, 179-191.

13 M. K. Joubert, M. Deshpande, J. Yang, H. Reynolds, C. Bryson, M. Fogg, M. P. Baker, J. Herskovitz, T. J. Goletz, L. Zhou, M. Moxness, G. C. Flynn, L. O. Narhi and V. Jawa, PLoS One, 2016, 11, e0159328.

14 D. M. Kennedy, A. W. Skillen and C. H. Self, Clin. Exp. Immunol., 1994, 98, 245-251.

15 R. Dolhofer, E. A. Siess and O. H. Wieland, Biol. Chem. Hoppe-Seyler, 1985, 366, 361-366.

16 ICH, ICH Topic Q 6 B Specifications: Test Procedures and Acceptance Criteria for Biotechnological/Biological Products, 1999.

17 European Medicines Agency, Committee for Medicinal Products for Human Use (CHMP) Guideline on similar biological medicinal products containing monoclonal antibodies-non-clinical and clinical issues, http://www.ema. europa.eu, (accessed 17 June 2019).

18 FDA, Development of Therapeutic Protein Biosimilars: Comparative Analytical Assessment and Other QualityRelated Considerations Guidance for Industry DRAFT GUIDANCE, https:/www.fda.gov/vaccines-blood-biologics/ guidance-compliance-regulatory-information-biologics/biologics-guidances, (accessed 17 June 2019).

19 N. Alt, T. Y. Zhang, P. Motchnik, R. Taticek, V. Quarmby, T. Schlothauer, H. Beck, T. Emrich and R. J. Harris, Biologicals, 2016, 44, 291-305.

20 B. Wei, K. Berning, C. Quan and Y. T. Zhang, $m A b s, 2017,9$, 586-594.

21 C. Quan, E. Alcala, I. Petkovska, D. Matthews, E. Canova-Davis, R. Taticek and S. Ma, Anal. Biochem., 2008, 373, 179-191.

22 L. J. Brady, A. Theresa Martinez and A. Balland, Anal. Chem., 2007, 79, 9403-9413. 
23 N. C. Dingari, G. L. Horowitz, J. W. Kang, R. R. Dasari and I. Barman, PLoS One, 2012, 7, e32406.

24 Y. Han, J. Chen, T. Pan and G. Liu, Chemom. Intell. Lab. Syst., 2015, 145, 84-92.

25 J. V. Glenn, J. R. Beattie, L. Barrett, N. Frizzell, S. R. Thorpe, M. E. Boulton, J. J. McGarvey and A. W. Stitt, FASEB J., 2007, 21, 3542-3552.

26 T. Pan, M. Li, J. Chen and H. Xue, J. Innovative Opt. Health Sci., 2014, 07, 1350060.

27 K. A. Esmonde-White, M. Cuellar, C. Uerpmann, B. Lenain and I. R. Lewis, Anal. Bioanal. Chem., 2017, 409, 637-649.

28 T. De Beer, A. Burggraeve, M. Fonteyne, L. Saerens, J. P. Remon and C. Vervaet, Int. J. Pharm., 2011, 417, 32-47.

29 J. Müller, K. Knop, M. Wirges and P. Kleinebudde, J. Pharm. Biomed. Anal., 2010, 53, 884-894.

30 H. F. Bunn, R. Shapiro, M. Mcmanus, L. Garrick, M. J. Mcdonald, P. M. Gallop, K. H. Gabbay, P. Bent and B. Hospital, J. Biol. Chem., 1979, 254, 3892-3898.
31 B. H. Shilton, R. L. Campbell and D. J. Walton, Eur. J. Biochem., 1993, 215, 567-572.

32 O. Anjos, M. G. Campos, P. C. Ruiz and P. Antunes, Food Chem., 2015, 169, 218-223.

33 K. J. Welsh, M. S. Kirkman and D. B. Sacks, Diabetes Care, 2016, 39, 1299-1306.

34 N. A. Ansari, A. S. Moinuddin and R. Ali, Dis. Markers, 2011, 30, 317-324.

35 M. E. Rubio-Ruiz, E. Diaz-Diaz, M. Cardenas-Leon, R. Arguelles-Medina, P. Sanchez-Canales, F. Larrea-Gallo, E. Soria-Castro and V. Guarner-Lans, Glycobiology, 2008, 18, 517-525.

36 B.-S. Lee, S. Krishnanchettiar, S. S. Lateef and S. Gupta, Int. J. Mass Spectrom., 2007, 260, 67-74.

37 A. Frolov and R. Hoffmann, Anal. Bioanal. Chem., 2010, 397, 2349-2356.

38 J. Juárez, P. Taboada and V. Mosquera, Biophys. J., 2009, 96, 2353-2370. 